\title{
EL PAPEL DEL JUEGO INFANTIL EN LA CULTURA: APUNTES Y APORTES PARA UNA ANTROPOLOGÍA DEL JUEGO
}

Xavier Ernesto Rodríguez Corea*

\begin{abstract}
Resumen
El presente artículo aborda el juego infantil, buscando explicar la relación entre este y la cultura. Además destaca su importancia y su relación con otros elementos como el espacio, las divisiones sociales por edad, sexo, y sus implicaciones entre las clases sociales.
\end{abstract}

Palabras claves: juegos infantiles, cultura, clases sociales

\begin{abstract}
The present article addresses infant games, trying to explain relation with culture. It also highlights its importance and its relationship with other elements such as space, social division by age and sex, and implications between social classes
\end{abstract}

Keywords: intantil games, culture, social classes

\section{Introducción}

E ser humano desde sus primeros pasos se ve enfrentado a la necesidad de reproducirse y asegurar su existencia, locuallovincula profundamente con las otras especies animales. Además de satisfacer sus necesidades fisiológicas, se encontró frente a la naturaleza y frente a otros humanos, tanto los de sus grupos como los otros --también estos extraños-buscado la supervivencia, alimentarse, reproducirse, crecer, comunicase, conocer y reconocerse. La cultura se perfila entonces, como una respuesta del ser humano ante la naturaleza y ante los otros. El juego es parte de esa necesidad de carácter social pero vinculado profundamente con la posibilidad de descubrir el cosmos, sus reglas y sus lógicas.

En términos del lenguaje coloquiales se entiende el juego como un elemento de la vida carente de seriedad. De hecho la seriedad es entendida como la antítesis del juego, sin embargo en términos científicos, la antropología pedagógica aborda el juego como un elemento de vital importancia para los procesos de sociales

\section{Material y métodos aplicados}

Para la elaboración de este artículo se recurrió a la búsqueda, selección y relación de teorías científicas a manera de discusión teórica que permita abordar la temática.

\footnotetext{
Docente-investigador. Departamento de Antropología. Facultad de Humanidades y Ciencia Jurídicas, Universidad Nacional Autónoma de Nicaragua-Managua. UNAN, Managua.
} 
La idea es tratar de recurrir en primera instancia a la teoría antropológica y también a la teoría de otras ciencias sociales como la pedagogía, las cuales aportan a la comprensión teórica del fenómeno.

También es importante mencionar que las reflexiones planteadas en este escrito, nacen de la experiencia del trabajo campo en diversos contextos, como parte la actividad académica docente en las asignaturas de antropología pedagógica, antropología del trabajo y antropología urbana. El trabajo de campo, independientemente de la temática que se desarrolle, aporta una serie de observaciones, reflexiones sobre el área temáticas señaladas la cual es posible tejer junto con la teoría existente, es así como nace y se desarrolla las ideas fuerzas que aquí se vierten.

Este articulo también se apoya en las investigaciones monográficas realizadas por estudiantes y coordinadas por el Departamento de Antropología de la Universidad Nacional Autónoma de Nicaragua (UNAN, Managua). En especial se han tomado en cuenta los trabajos desarrollados en el oriente y occidente de la zona del Pacifico del país. Estos trabajos monográficos están trabajados basados en la metodología etnográfica estructurado por los estudiantes al culminar sus estudios.

\section{Resultados y discusión}

Para entender mejor el juego en lo general y el juego infantil en lo particular, es necesario vincular algunas aportaciones epistemológicas que expliquen este fenómeno en toda su magnitud cultural. En otras palabras, necesitamos una episteme-ludico adecuado, partiendo de la categoría de cultura, pero haciendo la siguiente distinción: si bien es cierto, desde la antropología existen muchas posibilidades teóricas, también lo es, la posibilidad de plantear elementos que caractericen propuestas teóricas adecuadas al fenómeno. Para esto Gilberto Jiménez (2005) propone tres aportes para la aproximación a las diversas y siempre complejas categorías de cultura.

Según Jiménez, se pueden señalar tres aportes de las escuelas del pensamiento antropológico, distantes una de las otras en sus propuestas y temporalidad. Desde la propuesta evolucionista retoma la cultura como conjunto de costumbres formas o estilos de vida que caracterizan la vida de un pueblo. Este aporte rompe en su tiempo con la concepción de cultura como conocimiento, arte y ciencia, atribuidos únicamente a ciertos estratos sociales privilegiados.

Posteriormente, la escuela de cultura y personalidad entre otros, enfocan la cultura desde los modelos de comportamiento, más en concreto, a partir de los valores y los modelos normativos que fungen como marco referencial del comportamiento.

Finalmente, desde la antropología simbólica, la cual centra el análisis de la cultura desde el aspecto simbólico de esta. La delimitación a lo simbólico permite mayor eficacia teórica para bordar los fenómenos que la antropología trata de interpretar.

Este aporte de Jiménez resulta interesante al abordar el fenómeno del juego infantil, por el hecho que los tres aportes provienen de tres escuelas muy particulares en sus planteamientos. De manera que "estilos de vida", "modelos normativos" y "símbolo" conforman una triada que grosso modo deslumbra la magnitud del juego en la cultura. 
Por otra parte, la cultura desde la antropología implica también procesos de transmisión --aunque parezca obvio mencionarlo-- la cultura es aprendida, enseñada. Por consiguiente, en el proceso dialéctico de enseñanza-aprendizaje es trasformada.

Otro elemento importante a dilucidar es "el símbolo", el cual se presenta como capacidad humana relacionado directamente con la praxis cotidiana, naturaleza y grupo. El símbolo es ante todo metamórfico por su necesidad, es decir en su necesidad de cambiar constantemente, como la realidad social y el medio ambiente. El símbolo es capaz de dar sentido a elementos de la vida cotidiana que trasciendan, más allá del grupo y del tiempo, claro está, por su capacidad y necesidad de cambiar y re-significarse.

Por otro lado, la importancia pedagógica radica en la capacidad para sintetizar la praxis. De relacionarse y dar significado a aspectos de la vida cotidiana que el símbolo hace sobresalir sobre los otros, pues:

Si partimos del principio de que los símbolos no son sino representaciones de objetos de una naturaleza por otros de diferente naturaleza, no erramos al afirmar que el ser humano organiza su vida en una doble realidad: la objetiva y la simbólica (Claudio Malo.2006; pág 19)

De manera que esta doble realidad constituye "la realidad" que vivimos cotidianamente. Por otro lado, el aspecto lúdico se refiere a la posibilidad del desarrollo físico y mental que proporciona el juego, como parte de nuestra humanidad "homos ludens" (Morin, 1999). Por lo que el juego se convierte, como se ha apuntado anteriormente, en una actividad profundamente humana, en su significado y en su desarrollo histórico, pues desde sus primeros pasos la humanidad ha recurrido a los espacios y momentos lúdicos como complemento de otras actividades productiva "homo faber", conformando junto con otros aspectos, su racionalidad "homo sapiens"

En este sentido el juego debe ser distinguido de lo lúdico puesto que lo primero está integrado en lo segundo. El juego se perfila en la vida del ser humano como la posibilidad de aprender en un marco, contralado, lúdico y simbólico. Para el control están hechas las reglas mencionadas y las entendidas; lo lúdico se refiere a la posibilidad de diversión y distracción en ellos y lo simbólico en la posibilidad que tiene el juego para sintetizar la vida y desarrollar ideas, habilidades y destrezas mentales relacionadas con la realidad.

Así por ejemplo, en cualquier juego infantil es posible encontrar reglas claramente establecidas que permiten desarrollar el juego y aunque existen diversos niveles de estructuración, estas reglas están presentes incluso en aquellos juegos que parecieran anárquicos. Sin embargo, es importante mencionar que estas reglas entendidas socialmente, forman parte importante de la posibilidad del juego para desarrollar habilidades y divertirse, estableciendo ganadores, perdedores, premios y castigos entre otros. Es decir, no es la carencia de reglas o la cantidad de estas que hace el juego importante en términos culturales, sino la justa relación entre estos dos aspectos, la diversión y el control, pues ambos conforman un delicado equilibrio educativo-lúdico

Por otro lado Jean William Piaget Pisco (1989) hace un aporte especialmente significativo al análisis del juego en relación con el aprendizaje. Este autor lo aborda como proceso de aprendizaje en sí misma: 
(...) el juego es, primero que todo, simple asimilación funcional o reproductiva (...) que se explica por el proceso biológico según el cual todo órgano se desarrolla al funcionar (...) cada actividad mental -desde las elementales hasta las tendencias superiores tiene necesidad de desarrollarse de ser alimentada por un constante aporte exterior (pág. 123.)

De manera que el juego desarrolla significados sociales, pues si el símbolo es metamórfico, aquí no entendemos la forma, su aspecto físico sino lo social y en este caso cultural, por lo tanto el juego construye y de-construye. Es por eso que afirmamos que todos jugamos pero no todos jugamos lo mismo.

Debemos distinguir el juego como categoría general del juego infantil como aspecto específico, caracterizado por el sector atareo que lo practica. En nuestra sociedad existen límites bien claros sobre los tipos de juegos para adultos y para infantes.

\section{El patio y la calle, el parque, el lugar del juego}

El juego no se desarrolla en cualquier espacio, posee espacios preferenciales dados por las condiciones naturales e históricas. Tomando en cuenta el origen rural de la población nicaragüense, el patio sustituyo al campo. En el ámbito rural, el patio se extiende en amplias extensiones cercanas a la casa. En la medida en que el campo se ruraliza los patios se hacen más pequeños y limitados, sin embargo sigue siendo el lugar natural para el juego. En el patio se corre, se brinca, se ríe, se grita, en el interior de la casa se reposa, se habla, se escucha, de tal manera que el patio ocupa un lugar simbólico importante para el juego, pues aunque en otros espacios se desarrollan actividades lúdicas, es el patio el destinado para el juego infantil. El juego se desarrolla entre los primos y hermanos, el cual es permitido por el tamaño del espacio físico y de la unidad domestica.

Un ejemplo claro de este fenómeno lo encontramos en la monografía de Tania Lezama (2009), antropóloga quien en su trabajo de investigación titulado Territorialidad e identidad étnica en la comunidad el Jícaro San Dionisio Matagalpa documenta:

Dentro de la comunidad no hay parques ni centros deportivos en donde los jóvenes y niños puedan divertirse, para ello los patios de las viviendas y los predios baldíos son utilizados para practicar deportes tales como el béisbol y fútbol (pág. 31)

Por lo anterior, es posible animarnos desde el plano etnográfico a caracterizar los espacios para el juego en las comunidades rurales. En este trabajo queda planteada la necesidad de profundizar aún más el fenómeno del juego infantil rural

En los contextos más urbanizados, la calle se convierte en el espacio del juego, pues el patio ha llegado a extremos de limitación. La calle es el nuevo espacio para la recreación infantil, la casa ocupó el espacio del patio o la casa por las limitaciones urbanas, nació sin o con poco patio. También la familia urbana se ha reducido por lo que los primos y hermanos no son suficientes, los vecinos se convierten en los nuevos compañeros de juegos; en muchos sectores y a causa del desarrollo histórico particular, los vecinos también son primos o parientes pero no miembros de la unidad domestica residencial.

En otros contextos geográficos y culturales vendidos a nosotros y nosotras, por los 
medios de comunicación como primer mundo, el parque se convierte en un espacio primario de recreación. Sin embargo en nuestra realidad el parque en su devenir histórico no ha fungido como tal, en las ciudades y pueblos organizados con la lógica colonial, se convertía en un espacio público relacionado con el poder políticoreligioso. Pero en ningún momento es preponderante para la diversión infantil, más aun si este sector etario no pertenecía a la clase social dominante, excluida del centro del la ciudad. A esto es importante apuntarle los esfuerzos de algunas instituciones y grupos por proyectos urbanísticos que aproximen los centros a las poblaciones.

En los desarrollos urbanos posteriores (siglos XX, XXI) los espacios urbanos rara vez contaban con parques. En otros casos, luego de contar con estos espacios, desaparecían en su concepción original y eran sustituidos o modificados en sus usos, adquiriendo representaciones negativas o en el mejor de los casos permanecieron, pero por la forma en que crecía la urbe, quedaban cada vez más alejados de la población. En otras palabras, eran rebasados por la presión de la población. El parque nunca fue un lugar preponderante en la vida lúdica de la niñez nicaragüense, esto sin excluir diversas experiencias particularidades más que colectivas.

De manera que el espacio físico y su significado social tienen una relación estrecha con el juego en su papal educativo. El espacio es símbolo y el juego también, no se puede analizar uno lejos del otro. Bajo el sentido del lugar antropológico (Auge, 2000) la calle y el patio adquieren un sentido más allá de lo público y lo privado, se constituyen en lugares que dan cuenta de nuestra memoria colectiva

\section{El juguete}

El juguete podría expresar el aspecto más tangible del juego, aunque no todos ellos están acompañados por un elemento tangible. Cuando este aparece se convierte en portador de una gran cantidad de significados. En los juegos tradicionales nicaragüenses, los juguetes son un elemento importante de la infancia, no solo por su uso sino por su fabricación, ya que los juguetes tradicionales inician su vida, no con el comercio sino con la elaboración del mismo. El convertir una cuerda para usos agrícolas en un instrumento para saltar o un pedazo de madera para modelar un trompo, estamos frente a implicaciones que desarrollan habilidades sociales $y$ manuales, con implicaciones significativas en lo educativo y por lo tanto en lo cultural.

Contrario a la publicidad del mercado los juguetes tradicionales del pueblo nicaragüenses no se caracterizan por su complejidad o estética comercial, así por ejemplo es posible encontrar en las calles de los pueblos una patineta elaborada con tablas viejas y valieras descartadas, juguete que compite en su complejidad con cualquier patineta o patines que se comercian hoy en tiendas y que poco a poco desplazan a aquellos elaborados por el pueblo pobre que no accede a estos productos comerciales, los trompos, yoyos, bates, pelotas, guantes, muñecas y una innumerable lista de obras de arte elaborados en algunos casos por artesanos especializadas y en otro casos por niños, niñas, padres o madres como una extensión del juego infantil.

La elaboración de juguetes no solo toca el aspecto educativo pues aprender a elaborarlos forman parte de la trasmisión de conocimientos tradicionales en las poblaciones también trastoca el aspecto 
económico, en aquellas familiar artesanas que elaboran juguetes como su actividad laboral principal o emergente y en aquellas que ven alterada su lógica de consumo por la necesidades impuestas por la cultura de consumo capitalista dominante

\section{Todos jugamos pero no todos jugamos lo mismo}

Como se ha mencionado el juego infantil es una característica de la socialización humana sin embargo en el correspondiente análisis de clase aplicado a nuestra sociedad es importante señalar las distancias que existe entre este fenómeno entre las clases sociales burguesas y proletarias y los contextos urbanos y rurales, la primera división dada por el análisis marxista y la segunda por la distinción etnográfica de estos dos espacios económicos-culturales, esta concepción dual que es característica del pensamiento científico occidental es usado en este análisis con la conciencia que la realidad es compleja y podemos encontrar aspectos de la vida matizados por uno y otro elemento (sincronía) al mismo tiempo (diacronía) se hace esta anotación con la finalidad de evitar futuras lapidaciones teóricas

En la primera distinción referente a las clases sociales podemos apuntar en primer lugar que los sectores de la sociedad con cierto nivel de acceso al consumo comercial y que presentan una fuerte influencias de los medios de comunicación, los cuales como es de esperarse responden en su gran parte de su espectros a la lógica de acumulación capitalista neoliberal y por lo tanto proponen un modelo de vida acorde con este modelo económico, aunque esta influencia se presenta en todos los aspectos de la vida el juego infantil se desarrolla en este marco, los niños y niñas se les propone como lugar ideal de juego espacios propuestos por el comercio, centro comerciales, pizzerías, centros recreativos, esta propuesta es para algunos sectores una posibilidad, ocasional o solamente una lejana posibilidad, esto asociado directamente con las pasibilidades económicas de la familias, para estos sectores al juguete se compra, el lugar se alquila, el comercio se encarga del resto.

Por otra lado podemos mencionar los sectores populares que, aunque influenciados por los medios y la lógica de consumo propuestas por estos optan ya sea por la imposibilidad de acceder a los sectores de consumo o de forma consciente en apego a estilos de vida caracterizado por el aspectos tradicionales y populares, el juego es parte de la vida y aunque existen lugares ideales, en la realidad se juega en cualquier lugar y momento, aunque no existan las condiciones de seguridad, como los niños en las calles, para quienes la frontera entre juego y trabajo es muy móvil, pues se juega a trabajar y se juega mientras se trabaja, esto tanto en áreas rurales como urbanos, pero característico de los sectores populares, el juego el lugar para el juego se crean, se fabrican, se dan, no se compran o se venden.

\section{A manera de conclusión}

\section{Pistas para des-construir el juego y para construir una nueva sociedad}

Como hemos apuntado anteriormente, En términos científicos el juego no es inocente, pues acarrea consigo significados y significancias y por su importancia en la socialización primeras se convierten en parámetros para la vida, de manera que los juegos nos explican ¿cómo funciona el mundo? y nos reconstruyen estas ideas a medida que crecemos y seguimos jugando, pues aunque aquí nos hemos referido a 
el juego infantil, debemos recordar que el juego, juega un papel importante en lo largo y ancho de la vida.

Debemos distinguir que todo juego desarrolla al menos tres aspectos: físicomotor, emocional y socio-cultural, el primero se refiere a la posibilidad de locomoción, movimientos, destrezas de carácter físico, el segundo se refiere al aspecto afectivo-emocional que se desarrolla en el juego y el tercero referido a la cosmogónico o culturales, estos tres elementos que podrían ser mas según el enfoque científico, pueden ayudarnos a la desconstrucción de cada juego infantil.

\section{Referencias Bibliográficas}

Auge, M. (2000). Los "no lugares" espacios de anonimato. Una antropología de la sobre modernidad. Barcelona, España: Gedisa.

Jiménez, G.. (2005 ). Teoría y análisis de la cultura. vol 1. México: CONACULTA.

González, C. M. (2006). Arte y cultura popular. Centro Interamericano de Artesanías y Artes Populares. Azuay, Ecuador: Universidad del Azuay.

Lezama, T. M. (2009). Territorialidad $e$ identidad étnica en la comunidad el Jícaro San Dionisio Matagalpa. Managua: UNAN-Managua.

Morin, E. (1999). Los 7 saberes necesarios para la educacion del mañana. UNESCO, 12.

Piaget, J. (1989). La Formación de Símbolo en el niño. México: FCE. 